human chromosome 16. (Szepetowski P, Rochette J, Berquin P, Piussan C, Lathrop GM, Monaco AP. Familial infantile convulsions and parozysmal choreoathetosis: a new neurological syndrome linked to the pericentromeric region of human chromosome 16. Am I Hum Genet Oct 1997;61:889-898). (Reprints: Dr Anthony P Monaco, The Wellcome Trust Centre for Human Genetics, University of Oxford, Windmill Road, Headington, Oxford OX3 7BN, UK).

COMMENT. This study provides genetic evidence of an epileptic basis for paroxysmal choreoathetosis. Clinical features in common with epileptic seizures include paroxysmal attacks, tendency to remit, EEG epileptiform abnormalities, and response to antiepileptic drugs. A focus in the basal ganglia has been proposed for this syndrome. In differential diagnosis, choreoathetosis is a rare side effect of several AEDs. (Progress in Pediatric Neurology III, 1997;p157).

\title{
TEMPORAL LOBE EPILEPSY ETIOLOGICAL CLASSIFICATION
}

Clinical, EEG, and neuroimaging findings in a community-based cohort of 63 children with new-onset temporal lobe epilepsy (TLE) studied prospectively are reported from the Royal Children's Hospital, Melbourne, Australia. Three etiologically defined subgroups of TLE are proposed based on clinical history and MRI or CT findings: 1) Cryptogenic, with normal MRI and negative past history (54\%); 2) Hippocampal sclerosis, or previous illness (29\%); and 3) Developmental, malformation or tumor (16\%). Febrile seizures had preceded onset of TLE in 13 children, and bacterial meningitis in 4 . Neuroimaging revealed structural abnormalities of the temporal lobe in 24 (38\%); hippocampal sclerosis in $13(21 \%)$, tumor in 8 (13\%). Focal temporal EEG abnormalities were recorded in 19 of 24 with lesions on MRI or CT and in 27 of 39 with normal neuroimaging. Developmental, behavioral, or learning problems occurred in $38 \%$ of the cohort. Behavior problems included hyperactivity (14), aggressiveness (13), and rage attacks (5). (Harvey AS, Berkovic SF, Wrennall JA, Hopkins IJ. Temporal lobe epilepsy in childhood: Clinical, EEG, and neuroimaging findings and syndrome classification in a cohort with new-onset seizures. Neurology October 1997;49:960-968). (Reprints: Dr A Simon Harvey, Department of Neurology, Royal Children's Hospital, Parkville, Victoria 3052, Australia).

COMMENT. This extensive prospective study has provided a classification of temporal lobe epilepsy in childhood of value in prognosis and management. Temporal lobe epilepsy in childhood is classified etiogically in three groups: 1) cryptogenic, 2) hippocampal sclerosis, and 3) developmental. Children with cryptogenic seizures have a relatively good prognosis, whereas those with hippocampal sclerosis or developmental lesions are at risk of resistant seizures and psychological disabilities. For previous reports from the Melbourne, Australia group, the Cleveland Clinic experience, and that of the University of California, Los Angeles, see Progress in Pediatric Neurology II, 1994;pp78-79. Vol III, 1997;pp54-55.

SPECT-EEG correlations in temporal lobe epilepsy are reported from Yonsei University Coll Med, Severance Hospital, Seoul, Korea. (Lee BI, Lee JD, Kim JY, et al. Neurology Oct 1997;49:981-991). Interictal SPECT correctly lateralized $8 / 9$ patients with unitemporal epileptiform discharges and $5 / 10$ with bitemporal EEG discharges. Ictal SPECT was highly concordant with ictal EEG, but correctly lateralized the lesion in only $11 / 19$. 
In the Melbourne experience (Harvey et al. 1993), ictal SPECT lateralized lesions and correlated with EEG and MRI findings; it was superior to ictal EEG in localizing value in some. (PPN II, 1994;p79).

Mesial temporal spikes interfere with working memory in a report of 8 adult patients with depth electrodes implanted to evaluate intractable epilepsy, at Johns Hopkins Epilepsy Center, Baltimore, MD. (Krauss GL, Summerfield M, Brandt J, Breiter S, Ruchkin D. Neurology Oct 1997;49:975980). The greatest impairment of spatial and verbal recall coincided with left hippocampal spiking.

\section{FIRST TONIC-CLONIC SEIZURE: TREATMENT AND PROGNOSIS}

The outcome of immediate treatment (carbamazepine, phenytoin, phenobarbital, or sodium valproate) or delayed treatment after a second seizure, in two groups of patients followed for a minimum of 3 years, was evaluated in a multicenter, randomized, open trial reported by the "FIRST Group" from Segrate, Italy. Patients aged 2 years and older were included, 27\% $<16$ years, and $67 \% 16-60$ years. Only $52(24 \%)$ of 215 patients assigned to immediate treatment of the first tonic-clonic seizure had a recurrence during follow-up, whereas $85(42 \%)$ of 204 randomized to delayed treatment experienced a relapse. Remote etiological factors, a secondarily generalized seizure, and epileptiform EEG abnormalities increased the risk and frequency of relapse. Young to middle-age adults, and patients treated acutely with benzodiazepines had a lower risk of relapse. The probability of a 1 year remission was $20 \%$ greater in the immediate treatment group compared to those beginning treatment only after a relapse, but the difference was not significant. Patients treated after the first or second seizure had the same probability of remaining seizure-free for 1 or 2 years. (Musicco M, Beghi E, Solari A, Viani F, for the Rirst Seizure Trial Group (FIRST Group). Treatment of first tonic-clonic seizure does not improve the prognosis of epilepsy. Neurology Oct 1997;49:991-998). (Reprints: Dr Massimo Musicco, Istituto di Tecnologie Biomediche Avanzate, Consiglio Nazionale delle Ricerche, Via Filli Cervi 93, 20090 Segrate (MI), Italy).

COMMENT. The risk of seizure recurrence after a first unprovoked seizure is reduced $50 \%$ by immediate initiation of antiepileptic therapy. The lessening of patient and parent anxiety and the avoidance of possible complications of a second seizure by early treatment must be weighed against the risks of adverse effects of medications. Since more than 50\% of untreated patients are seizure-free at the end of a 1 to 2 year follow-up period, many authorities elect to defer therapy pending a seizure recurrence. To prevent the unnecessary AED exposure of patients with a low recurrence risk, an individually determined therapeutic approach is most appropriate, having regard to risk factors such as age, etiological factors, and EEG findings. Reliance on the use of rectal diazepam to limit severity and duration of acute seizure recurrence should favor the delayed therapy approach.

A previous prospective study and report from Montefiore Medical Center, New York, involved 407 children followed for a mean of 6 years. The risk of seizure recurrence after a first unprovoked seizure was $37 \%$ at 2 years and $42 \%$ at 5 years. The authors emphasize the improved quality of life as justification for rational therapy and seizure control. (Shinnar et al. 1996; see Progress in Pediatric Neurology III, 1997;pp88-89). 